\title{
Indoor localization for autonomous mobile robot based on passive RFID
}

\author{
Sunhong Park \\ Department of Applied physics \\ Waseda University \\ 3-4-1 Okubo Shinjuku Tokyo, JAPAN \\ paku@shalab.phys.waseda.ac.jp
}

\author{
Shuji Hashimoto \\ Department of Applied Physics \\ Waseda University \\ 3-4-1 Okubo Shinjuku Tokyo, JAPAN \\ shuji@waseda.jp
}

\begin{abstract}
In this paper, we propose a novel method using read-time of IC tags to reduce the localization error of an RFID navigation system. In order to reduce localization error, many approaches have been introduced. However, they do not deal with the problems that arise when the antenna can read at most one tag at any given moment. Using this, our passive RFID system is able to estimate the robot's location and orientation more accurately without the use of external sensors or a vision system. The experimental results show that the proposed method effectively estimates both the location and the pose of a mobile robot during navigation. We present results of trajectories of the robot in navigation and compare them with a generally utilized RFID algorithm.
\end{abstract}

Index Terms - Localization, read-time model, passive RFID, autonomous mobile robot.

\section{INTRODUCTION}

As mobile robots are becoming autonomous system gradually, the localization capability becomes a fundamental problem. It is easy to perform assigned tasks more safely if a mobile robot is able to move based on estimating accurate localization. There are two kinds of localization methods. One is relative localization, including dead reckoning and inertial navigation. The other is absolute localization, including RFID, active beacon, landmark and map-based localization. The existing dead reckoning (DR) approaches [1], [2], which enables it to estimate the total distance traveled from a stating point. However, dead reckoning is problematic in that the estimation error accumulates over time since no external reference signals are employed for correction. In order to improve the accuracy of localization, many approaches combine external sensors; such as cameras, beacon, laser range finders, sonar, GPS etc. Makela et al. [3] studied the integration of dead reckoning and visual landmark recognition methods for the navigation of outdoor mobile robots. Stella et al. [4] proposed a cooperation strategy between odometry and visual self-location method. Hahnel et al.[5] built 3D models with a laser scanner mounted vertically on mobile robot

This research was supported in part by WABOT HOUSE project funded by Gifu prefecture and the Global COE (Centers of Excellence) Program, "Global Robot Academia", Waseda University. This research was also supported by the Project for Strategic Development of Advanced Robotics Elemental technologies (NEDO: 06002090) and a Waseda University Grant for Special Research Projects (2007B-168). equipped with a horizontal 2D localization system. Moravec et al. [6] used evidence grids in 3D space based on stereo vision. There are also methods to revise the accumulated errors by external sensors using landmarks [7]-[9]. These methods estimate the location and pose of the robot from the observation of landmarks established in environment.

However, the landmarks are not always observable due to the influence of ambient light and shielding obstacles. Additionally, ultrasonic systems, like the Active Bat localization systems [10], use an ultrasound time-of-flight measurement technique to provide location information. In order to determine pose information of a mobile robot, many approaches have used external sensors such as cameras providing $2 \mathrm{D}$ and $3 \mathrm{D}$ information about the environment [11], [12].

In case of RFID systems, it can provide location information of a robot, it is difficult to accurately calculate location of the robot due to the nature of the antenna, which can only detect if a IC tag is present or not. Therefore, a mobile robot normally requires other sensors for estimating its precise location and pose.

In this paper, we propose new method for localization for a mobile robot localization using read-time of antenna by RFID system. In order to examine its validity, we implemented it on an autonomous mobile robot. We do not utilize multiple IC tags for reducing localization error because of the increased number of required tags. In general, a pair of antennas is necessary to identify the orientation of the mobile robot. However, we are able to estimate the orientation using the relation between the previous and current IC tags. Using our approach, we can acquire robust environment information. The IC tags are small and low cost, making them easily installed in a human living environment. For example, they can even be installed under carpets or other flooring materials, since unlike barcodes they are identified by radio frequency. Moreover, IC tags are durable against dirt, jitter, vibration and wear.

This paper is organized as follows. After discussing related work, we will present a brief overview of the autonomous mobile robot (UBIRO) in Section III and RFID system in Section IV. In section $\mathrm{V}$, the localization and pose estimation algorithms are described. We performed a series of experiments with the developed algorithms, the results of which are presented in Section VI. Finally, Conclusions and future work are described in Section VII. 


\section{RELATED WORKS}

Radio Frequency Identification (RFID) technology [13] has been utilized in the field of mobile robotics. Recent approaches in the field of mobile robot navigation have evidenced an increasing interest in the emerging RFID technology as promising alternative technique due to ease of use, flexibility, and low cost. Matsumoto et al. [14] reported a traveling system that uses RFID tags as external assistance to determine robot's position and direction. Khubitz et al.[15] presented a navigation system that uses RFID tags as artificial landmarks. The tags have information of global position( $\mathrm{x}, \mathrm{y}$, and $\mathrm{z}$ ), environment class, environment position, and further optional data. Hahnel[16] utilized RFID to improve localization of mobile robots that is equipped with a pair of RFID antennas and persons in their environment. Lionel et al. [17] presented LANDMARC, a location scanning prototype system that used active RFID for locating objects inside buildings. Seo et al.[18] suggest the sensor model of the RFID tag to reduce the robot's localization error applying the Markov localization and the Kalman filter localization algorithms. Han et al. [19] present localization scheme for an indoor mobile robot using RFID system. They adopted triangular pattern of arranging the RFID tags on the floor to reduce the estimation error of the conventional square pattern. Takehiro[20] proposed a location estimation system using UHF band RFID, with tags deployed on the ceiling. However, such an approach suffers from a variety of obstacles such as lighting fixtures, piping, and ventilation ducts. Most of mentioned above approaches was utilizing multiple RFID tags under RFID reader or adopting multiple RFID reader. Chon et al.[21] used an RFID system along with GPS and a gyroscope to produce highly accurate location information. They installed active IC tags which stored accurate location information on roads. Yamano et al.[22] proposed a method that performs self-localization with an RFID system using Support Vector Machines. In RFID technology, there is little research on both localization and pose estimation of a robot. Matsumoto et al. [23] combined RFID and GPS to estimate both localization and pose of robot. In these researches, generally only the location is estimated with the RFID system, while pose estimation is performed by other sensors. Additionally, some methods which use active IC tags require maintenance by humans, such as periodical battery exchange. Contrary to the mentioned above methods, we have proposed a method that is able to estimate localization and pose of a robot while navigating [24], [25]. Tsukiyama[26] developed a navigation system for mobile robots using RFID tags, however, their system assumed perfect signal reception and measurement and do not deal with uncertainty. On the other hand, we proposed a method that, by using an anti-collision function, is able to read plural tags at the same time [27]. From this experimental result, we could estimate a more precise location and pose estimation of the robot. However, it is not economical because of the increased number of required tags.

\section{MOBILE ROBOT}

In this chapter, we describe the robot system named UBIRO (UBIquitous RObot) used in the experiment. UBIRO consists of three main parts; the PC for control, the RFID system for obtaining the location information of robot, and the mobile base for navigation. UBIRO was developed based on an electric wheelchair [EMC-230] for the elderly and disabled persons as a mobility device. The PC mounted on the electric wheelchair acts as a central controller that handles information of IC tags from the RFID system and sends command to mobile base in order to reach the goal. The RFID system reads IC tags on the floor which allows the PC to roughly deduce the location and pose of the robot based on the proposed algorithms. Finally, the mobile base is controlled by the PC based on calculations result from information provided by the RFID system. UBIRO has external sensors such as distance sensors and touch sensors (bumper switches) for detection of obstacles. The robot's front wheels are free rotating casters, causing some instability when moving. UBIRO is shown in Fig. 1.

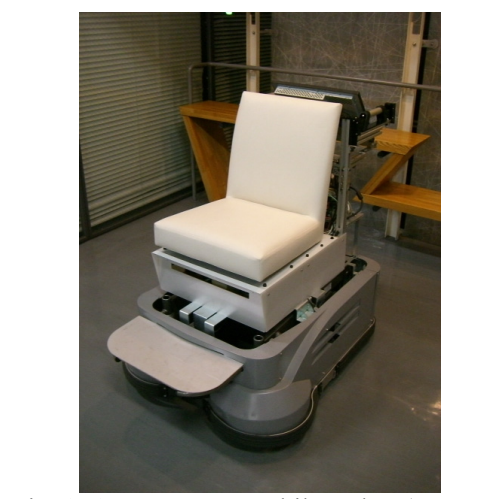

Fig. 1 Autonomous Mobile Robot (UBIRO)

\section{RFID SYSTEM}

In general, RFID systems consist of a reader, an antenna, IC tags and software such as drivers and middleware. The main function of the RFID system is to retrieve information (serial number) from an IC tag (also known as transponder) and to send it to the PC. An antenna is sometimes regarded as a separate part of an RFID system. The communication between an antenna and IC tags uses inductive coupling and each has a coil. The energy is transferred from an antenna to IC tags by means of mutual inductance between the two circuits. The frequency for the RFID system is $13.56 \mathrm{MHz}$.

A. Reader

In the RFID system, we adopted the compact and inexpensive RFID reader (Midrange Reader Module) made by Texas instrument. It operates at a frequency of $13.56 \mathrm{MHz}$ and handles all RF and digital functions in order to communicate with Tag-it HF, Tag-it HF-I and all other ISO 15693 devices. Moreover, it has an anti-collision function that is makes it able to read plural IC tags simultaneously.

B. Antenna 
The size of the antenna in the RFID system must be adjusted in accordance with the distance between IC tags. We constructed an experimental circular antenna that is able to detect IC tags on the $13.56 \mathrm{MHz}$ frequency band by using the S6350 Midrange Reader Module (Texas instruments). It is possible to read IC tags within $6 \mathrm{~cm}$ under the antenna. Thus, we assume that the IC tags are laid on the floor where the robot explores, attach the antenna to the underside of the mobile base, keeping the distance from the antenna to the floor approximately $5 \mathrm{~cm}$. The antenna is able to read IC tags which exist within about $17 \mathrm{~cm}$ from the center of the antenna.

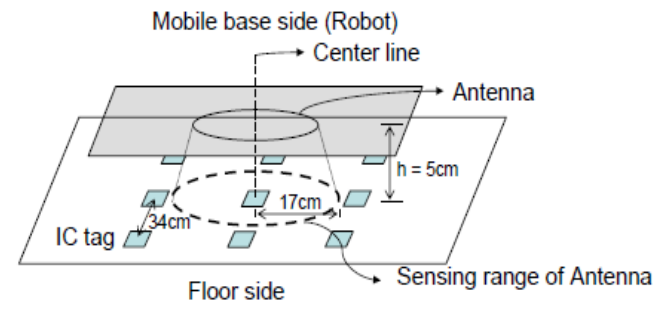

Fig. 2 Scanning range of the RFID antenna

\section{IC tag}

Generally, IC tags for RFID systems can be classified as one of three types; passive, semi-passive (also known as semiactive), and active. In consideration of no maintenance, cost, and indoor environment, we adopted the passive tags. Each IC tag is programmed to send a serial number that is related to the two-dimensional coordinate of absolute position of the tag in a database stored on the robot. Also, the IC tag is recognized only when it stays within the reception area of the antenna for more than $50 \mu \mathrm{s}$.

\section{IC Tag Distribution Patterns}

There are two IC tag arrangement patterns; random and the regular distribution. In case of the regular distribution pattern; Grid pattern and the triangulation pattern, the robot can sense at least one IC tag at any location. Although we must arrange IC tags maintaining the regular distance and pattern in environment, it is easy to get the location information without an additional special algorithm. In this paper, we use the regular distribution method and arrange the IC tags in the square grid-like pattern.

\section{LOCALIZATION With PASSIVE RFID System}

\section{A. Location recognition of mobile robot}

In general, the RFID system acquires the location information when the antenna detects an IC tag. If the antenna detects multiple IC tags as $\operatorname{Tag} 1\left(x_{1}, y_{1}\right), \operatorname{Tag} 2\left(x_{2}, y_{2}\right) \cdots$ $\operatorname{Tagn}\left(x_{n}, y_{n}\right)$,we are able to acquire the location of the mobile robot using equations (1), (2).

$$
\begin{aligned}
& x_{\text {estimation }}=\frac{\left\{x_{1}+x_{2}+\cdots x_{n}\right\}}{n} \\
& y_{\text {estimation }}=\frac{\left\{y_{1}+y_{2}+\cdots y_{n}\right\}}{n}
\end{aligned}
$$

In these equations, $n$ represents the number of IC tags detected by the antenna and $\left(x_{1}, y_{1}\right),\left(x_{2}, y_{2}\right) \cdots\left(x_{n}, y_{n}\right)$ represents the coordinates of those IC tags. In the event only a single IC tag is read, the mobile robot takes the $\left(x_{i}, y_{j}\right)$ coordinates as location of the mobile robot. In other words, the localization accuracy using the RFID system depends on the distance between the IC tags and their distribution pattern.

B. Localization

In any RFID system, error will persist without enough IC tags on the floor. However, simply increasing the number of tags is impractical due to the increasing cost. In light of this, while most researches involve using the center point of a number of tags read simultaneously, we use an arrangement and density that ensure only a single tag can be read at any one time.

In general, the polar coordinates $(r, \theta)$ are defined in terms of Cartesian coordinates $(x, y)$ by,

$$
\begin{aligned}
& x=r \cos \theta \\
& y=r \sin \theta \\
& r=\sqrt{x^{2}+y^{2}} \\
& \theta=\tan ^{-1}\left(\frac{y}{x}\right)
\end{aligned}
$$

where, $r$ is the radial distance from the origin, and $\theta$ is the counter clockwise angle from the positive $\mathrm{X}$-axis.

When the robot detects a new IC tag $Q\left(x_{2}, y_{2}\right)$ as shown in Fig.3, we can calculate robot's location as $P\left(x_{\text {current },} y_{\text {current }}\right)$ in IC tags field like equations (7), (8) using above equations.

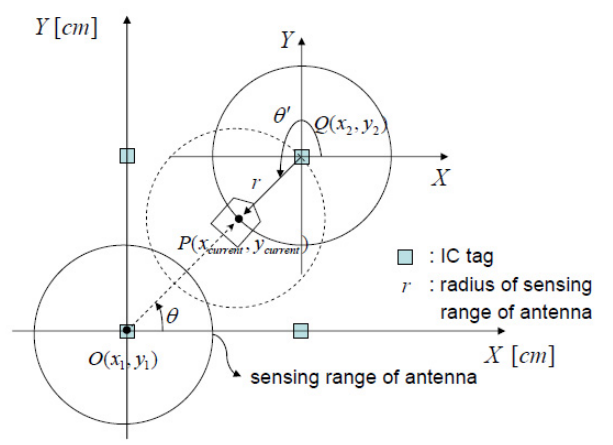

Fig. 3 Polar coordinate at $\mathrm{P}$

$$
\begin{aligned}
& x_{\text {current }}=r \cos \theta^{\prime}+x_{2} \\
& y_{\text {current }}=r \sin \theta^{\prime}+y_{2}
\end{aligned}
$$

Radius $r$ is $17 \mathrm{~cm}$, the same as the radius of the scanning range of the antenna. Let angle ( $\left.\theta^{\prime}\right)$ denote an incident angle against the newly detected IC tag. The equations (7), (8) are also used in the procedure for navigation.

We calculate the rotation angle for navigation to the goal by the following method. Firstly, the robot starts to move 
without considering the initial pose. The orientation of the goal, $\theta_{\text {start } \rightarrow \text { goal }}$, is derived from the initial and goal locations using equation (9).

$$
\theta_{\text {start } \rightarrow \text { goal }}=\arctan \left(\frac{y_{\text {goal }}-y_{\text {init }}}{x_{\text {goal }}-x_{\text {init }}}\right)
$$

In this equation, $\left(x_{\text {goal }}, y_{\text {goal }}\right)$ represents the coordinate of the goal location. Likewise, $\left(x_{\text {init }}, y_{\text {init }}\right)$ represents the coordinate of the start location. The robot obtains the coordinates of new IC tags from the antenna when it moves. We obtain the pose of the robot: $\left(\theta_{\text {previous } \rightarrow \text { current }}\right)$ using the equation (10).

$\theta_{\text {previous } \rightarrow \text { current }}=\arctan \left(\frac{y_{\text {current }}-y_{\text {previous }}}{x_{\text {current }}-x_{\text {previous }}}\right)+\theta_{\text {error }}$

,where $\left(x_{\text {previous }}, y_{\text {previous }}\right)$ and $\left(x_{\text {current }}, y_{\text {current }}\right)$ denote the coordinates of the location scanned previous and current, respectively, and $\theta_{\text {error }}$ represents the difference between the theoretical and physical location of the robot.

According to equation (11), we obtain the angle $\left(\theta_{\text {current } \rightarrow \text { goal }}\right)$ between the current location.

$$
\theta_{\text {current } \rightarrow \text { goal }}=\arctan \left(\frac{y_{\text {goal }}-y_{\text {current }}}{x_{\text {goal }}-x_{\text {current }}}\right)
$$

From the above equation (10) and (11), the rotation angle $\left(\theta_{\text {rotation }}\right)$ toward the goal is provided by the equation (12). The current location and orientation are updated in real time.

$$
\theta_{\text {rotation }}=\theta_{\text {previous } \rightarrow \text { current }}-\theta_{\text {current } \rightarrow \text { goal }}
$$

There are three kinds of movement; rotation (left / right) and forward movement of the robot according to the value of $\theta_{\text {rotation }}$. The forward movement speed of the robot is about $12.2 \mathrm{~cm} / \mathrm{s}$ in the experiment. The antenna reads new IC tags at an interval of $0.1 \mathrm{~s}$ and the robot rotates according to the calculated rotation angles periodically.

\section{Localization Error}

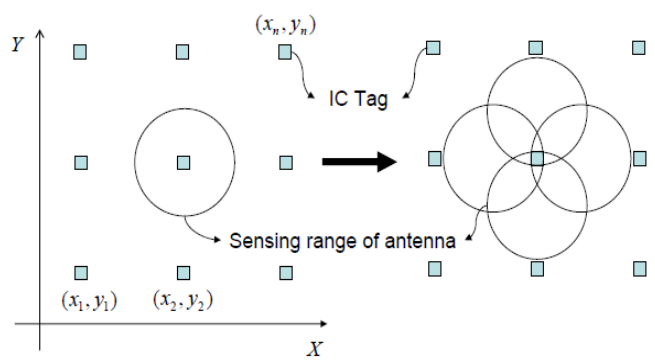

Fig. 4 Localization error of the RFID antenna

As shown in Fig.4 the localization error based on the RFID system is proportional to the scanning range of the antenna. Hence, many researches have utilized external sensors or other methods, such as Dead Reckoning (DR), vision systems, and sonar in order to reduce the localization error.

In using the RFID system, some researches propose methods which change the arrangement and raise the density of IC tags. However, the system cost is also increased because of the increased number of IC tags. Moreover, a problem with detection collisions arises as sometimes the antenna senses more IC tags at once than it can handle.

D. Read-time model of Antenna

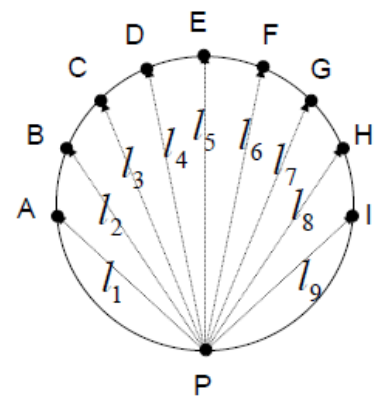

Fig.5 Read-time model

As shown in Fig.5, we propose a read-time model to reduce localization error in an RFID system. Read-time is defined as the length of time the antenna recognizes and reads a given tag, i.e. the amount of time a tag is in the scanning area. Let us assume that the mobile robot passed from $P$ to $A \sim I$ and that $l_{1}, l_{2} \cdots l_{9}$ represent the length when the mobile robot passes each $P A, P B \cdots P I$, respectively. Accordingly, the mobile robot is able to acquire different read-time measurements for each length. In other words, the read-time of is proportional to each length $l_{1}, l_{2} \cdots l_{9}$. Table I illustrates estimated read-times based on the average speed of the mobile robot, approximately $12.2 \mathrm{~cm} / \mathrm{s}$.

TABLE I

THE ESTIMATED READ-TIME IN EACH LENGTH

\begin{tabular}{|c|c|c|}
\hline Length & read-time [sec] & Angle [degree] \\
\hline PE & $2.6 \sim 2.8$ & 90 \\
\hline PD, PF & $2.2 \sim 2.6$ & $70 \sim 89$ \\
\hline PC, PG & $1.8 \sim 2.0$ & $60 \sim 70$ \\
\hline PB, PH & $1.1 \sim 1.2$ & $50 \sim 60$ \\
\hline PA, PI & $0.8 \sim 1.0$ & $40 \sim 50$ \\
\hline
\end{tabular}

E. Reduction of localization error

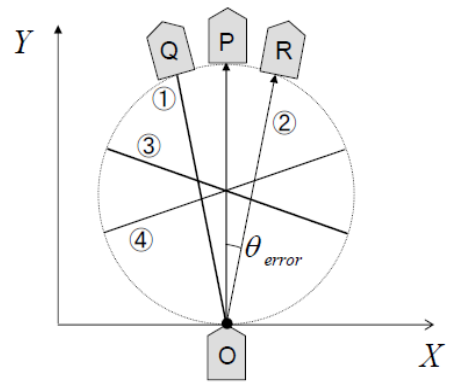

Fig.6 Example of reduction of localization error 
In proposed sensing model, some read-times are nondiscrete (1) (4), as shown in Fig.6. From (1) (4), we select the appropriate read-time based on previous pose of robot.

Let us assume that the center of the mobile robot passed through OP. However, the mobile robot may appear to pass through OR or OQ, because our prototype robot "UBIRO" occasionally arches because the front wheels are free casters. $\theta_{\text {error }}$ represents the difference in angle between calculated route $(\mathrm{OP})$ and actual route $(\mathrm{OR})$ as shown in Fig.5. We obtain $\theta_{\text {error }}$ when the mobile robot passed route (2) or (1) by choosing the path most likely taken based on the last turn the robot executed. In order to reduce localization error, the mobile robot gets feedback $\theta_{\text {error }}$ using the read-time after every step in navigation. The value of $\theta_{\text {error }}$ is used in equation (10).

\section{EXPERIMENTS AND RESULTS}

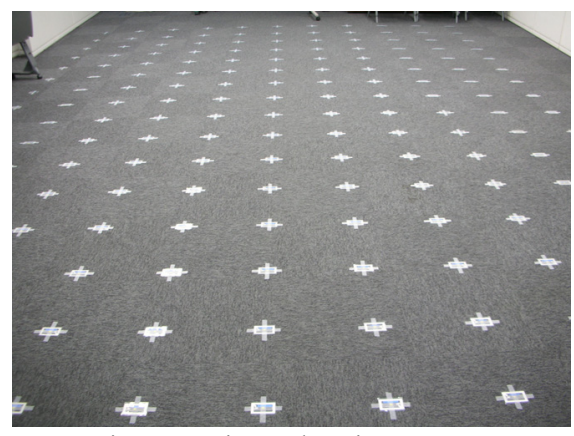

Fig.7 Experimental environment

In order to evaluate the feasibility of the proposed localization method using read-time of RFID system for mobile robot, we conducted experiments under various initial pose of the mobile robot. The experimental environment is shown in Fig.7. We assumed the environment as an indoor field where the IC tags were deployed. The 198 passive IC tags were laid on the floor in a grid-like pattern over an area measuring $420 \mathrm{~cm} \times 620 \mathrm{~cm}$, with a spacing of $34 \mathrm{~cm}$. There is no obstacle on the floor. The robot stops navigating when the antenna detects the IC tag of the goal area(within $34 \mathrm{~cm} \times$ $34 \mathrm{~cm}$ ). From various initial pose of mobile robot in navigation, Fig. 8 shows the result when the initial pose was 0 degree. We compared with the conventional method [23] without $\theta_{\text {error }}$ correction and the proposed method. The conventional method deals with localization problem in navigation. It was also one-to-one correspondence between the antenna and IC tag. The robot stops and ends navigation in case that the antenna detected the IC tag of the goal.

In general, conventional method acquired an intermediate location of the IC regardless of pose of robot which detected IC tag. On the other hand, we proposed new method using read-time of IC tag that could measure more accurately localization and pose of robot in real time. Table II illustrates localization error between the conventional method and proposed method. While localization error in the proposed method has $6.0 \mathrm{~cm}$ at $\mathrm{X}$-axis and $5.3 \mathrm{~cm}$ at $\mathrm{Y}$-axis in the average, conventional method has $17 \mathrm{~cm}$ at $\mathrm{X}-\mathrm{Y}$ coordinates. Furthermore, the total time of navigation using proposed method $(73 \mathrm{sec}$.) is shorter than conventional method $(85 \mathrm{sec}$.).

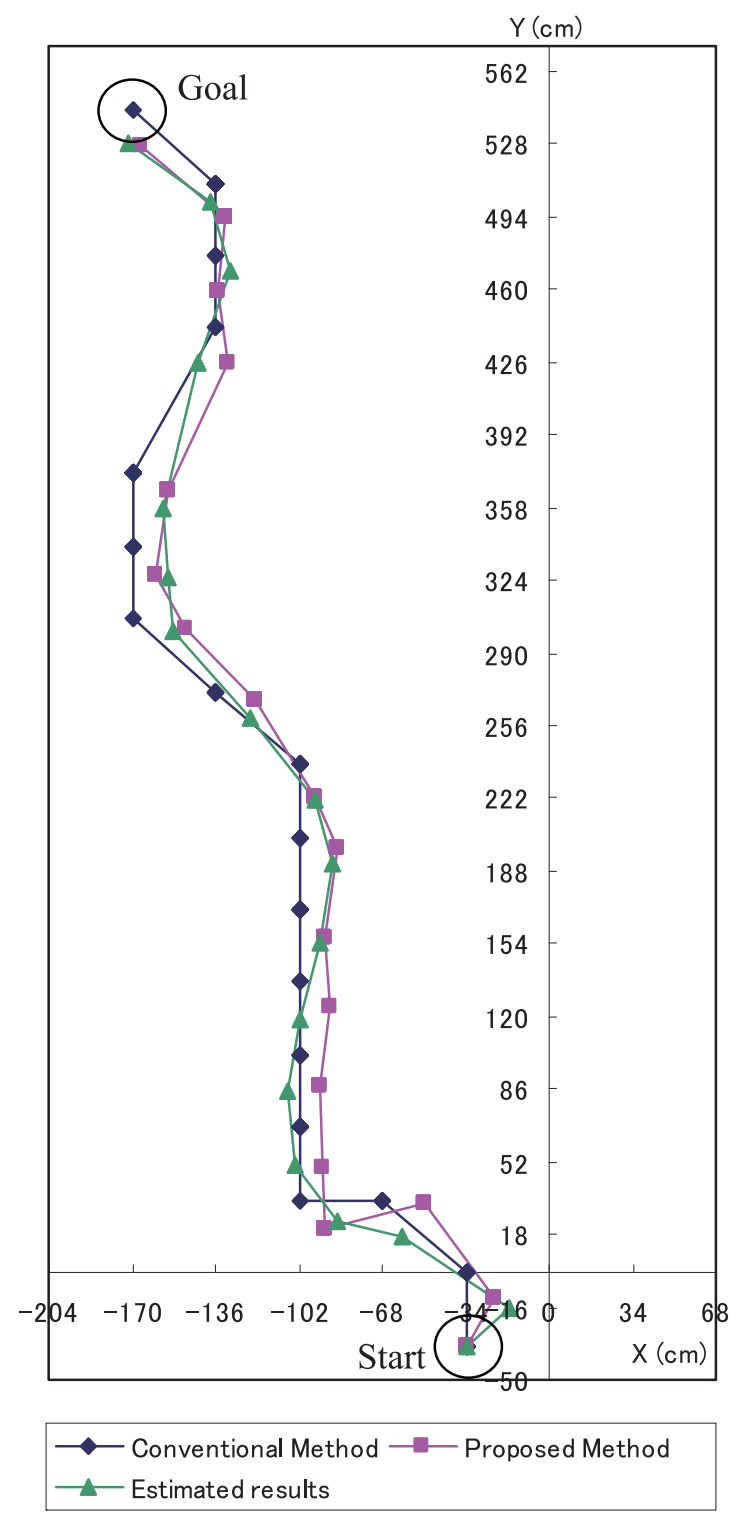

Fig.8 Path trajectories under initial pose 0 degree

TABLE II

COMPARISON OF LOCALIZATION METHODS

\begin{tabular}{|c|c|c|}
\hline Localization error & X-axis $[\mathrm{cm}]$ & Y-axis $[\mathrm{cm}]$ \\
\hline Conventional Method & 17.0 & 17.0 \\
\hline Proposed Method & 6.0 & 5.3 \\
\hline
\end{tabular}

\section{CONCLUSIONS}

In this paper, we propose a novel method using read-time model of IC tags to reduce the localization error. We examined the efficiency of the proposed method through indoor navigation experiments with the mobile robot. After 
examining the recorded trajectories, we can reduce the localization error without the use of external sensors or a vision system in order to modify the location of the robot. The IC tags can be identified robustly despite of any covers, jitter, dirt, wear or vibration, and so we conclude that the IC-taggedroom is a feasible option for real life applications.

We believe that our approach would be adequate for use in hospitals or nursing facilities for elderly and disabled people. In any navigation system using RFID, reliable detection of RFID tags is important for accuracy in estimating the location of the robot. For this reason, we set the speed of the mobile robot to about $12.2 \mathrm{~cm} / \mathrm{sec}$. The speed of robots using RFID systems depend on the read rate of RFID reader. If it is possible for the RFID reader to detect tags more quickly, the speed of the robot can be raised. In the near future, we are going to continue experimenting with different placing patterns and distances between the IC tags. We plan on redesigning the front wheels of the robot to allow us more precise motion control.

\section{REFERENCES}

[1] A. Kelly, "General solution for linearized systematic error propagation in vehicle odometry," in Proceedings of the IEEE/RSJ International Conference on Robotics and Systems(IROS), pp.1938-1945, 2001.

[2] J.L.Crowley, "Control of Displacements and Rotation in a Robot Vehicle," in Proceeding of the IEEE International Conference on Robotics and Automation, 1989.

[3] Makela, H.; Koskinen, K., "Navigation of outdoor mobile robots using dead reckoning and visually detected landmarks," Advanced Robotics, 1991. 'Robots in Unstructured Environments', 91 ICAR., pp.1051 - 1056, vol.2, 1991.

[4] Stella, E.; Lovergine, F.P.; Caponetti, L.; Distante, A., "Mobile robot navigation using vision and odometry," Intelligent Vehicles '94 Symposium, pp.417-422, 1994.

[5] Dirk Hahnel, Wolfram Burgard, and Sebastian Thrun, "Learning compact $3 \mathrm{~d}$ models of indoor and outdoor environments with a mobile robot," In the fourth European workshop on advanced mobile robots (EUROBOT'01), pp. 91-98, 2001.

[6] Hans P.Moravec, "Robot spatial perception by stereoscopic vision and 3d evidence grids," Technical report, CMU technical report, CMU-RITR-96-34, 1996.

[7] C. Drocourt, L. Delahoche, C. Pegard, and A.Clerentin, "Mobile robot localization based on an omni directional stereoscopic vision perception system," Proc. of Int. Conf. on Robotics and Automation, pp. 1329-1334, 1999.

[8] Akira Motomura, Takeshi Matsuoka, Tsutomu Hasegawa, Ryo Kurazume, "Real-Time Self-Localization Method by Using Measurements of Directions of Two Landmarks and Dead Reckoning," Journal of the Robotics Society of Japan, Vol.23, No.3,pp39-48, 2005.

[9] Margrit Betke and Leonid Gurvits, "Mobile Robot Localization Using Landmarks," IEEE Transactions on robotics and automation, vol.13, No.2, 1997.

[10] A. Harter, A. Hopper, P. Steggles, A. Ward, and P. Webster,'The anatomy of a context-aware application," Proceedings of the $5^{\text {th }}$ Annual ACM/IEEE International Conference on Mobile Computing and Networking (Mobicom 1999), pp.59-68, 1999.

[11] F.Lu and E.Milios, "Robot pose estimation in unknown environments by matching $2 \mathrm{~d}$ range scans," Journal of Intelligent and Robotic systems, vol.18, pp.249-275, 1997.

[12] S.Hakim, P.Boulanger, and F.Blais, "A mobile system for indoors 3-d mapping and positioning," Proc. of the $4^{\text {th }}$ Conference on Optical 3-D Measurement Techniques, 1997.
[13] Klaus Finkenzeller. RFID Handbook: Radio-Frequency Identification Fundamentals and Applications. Wiley, New York, 2000.

[14] Matsumoto, O.; Komoriya, K.; Hatase, T.; Nishimura, H.; Tsutomu Hatase; Hideki Nishimura, "Autonomous Traveling Control of the "TAO Aicle" Intelligent Wheelchair," Intelligent Robots and Systems, IEEE/RSJ International Conference, pp.4322 - 4327, 2006.

[15] Kubitz, O.; Berger, M.O.; Perlick, M.; Dumoulin, R , "Application of radio frequency identification devices to support navigation of autonomous mobile robots," Vehicular Technology Conference, vol.1, pp.126-130, 1997.

[16] Hahnel, D.; Burgard, W.; Fox, D.; Fishkin, K.; Philipose, M , "Mapping and localization with RFID technology," Proceedings of ICRA'04, vol.1, pp.1015-1020, 2004.

[17] Lionel M.NI, Yunhao Liu, Yiu Cho Lau, Abhishek P.Patil, "LANDMARC:Indoor Location Scanning Using Active RFID," Wireless Networks, Vol.10, No.6, pp.701-710, 2004.

[18] Dae-Sung Seo, Daeheui Won, Gwang-Woong Yang, Moo-Sung Choi, SangJoo Kwon, and Joon Woo Park, "A Probabilistic Approach for Mobile Robot localization under RFID Tag Infrastructures," 2005 International Conference on Control, Automation, and Systems (ICCAS 2005), pp 1797-1801, 2005.

[19] Soonshin Han, HyungSoo Lim, JangMyung Lee, "An Efficient Localization Scheme for a Differential-Driving Mobile Robot Based on RFID System," Industrial Electronics, IEEE Transactions on Volume 54, Issue 6, pp.3362-3369, 2007.

[20] Shiraishi T., Komuro N., Ueda H., Kasai H. Tsuboi T.,"Indoor Location Estimation Technique using UHF band RFID," Proceedings of the ICOIN 2008 international conference on information networking, pp.1-5, 2008.

[21] Hae Don Chon, Sibum Jun, Heejae Jung, Sang Won An,"Using RFID for Accurate Positioning," Journal of Global Positioning Systems, Vol.3, No. 1-2, pp.32-39, 2004.

[22] K. Yamano, K. Tanaka, M. Hirayama, E. Kondo, Y. Kimuro and M. Matsumoto, "Self-localization of mobile robots with RFID system by using support vector machine," Proceedings of the IEEE/RSJ International Conference on Intelligent Robots and systems, pp.37563761, 2004.

[23] Osamu Matsumoto, et. al, "Autonomous Traveling Control of the "TAO Aicle" Intelligent Wheelchair," Proceeding of the 2006 IEEE/RSJ international conference on Intelligent Robots and Systems, pp. 43224327, 2006.

[24] Sunhong Park, Ryo Saegusa, and Shuji Hashimoto, "Autonomous navigation of a mobile robot based on passive RFID," $16^{\text {th }}$ IEEE International Conference on Robot \& Human Interactive Communication (IEEE ROMAN2007), pp.218-223, 2007.

[25] Sunhong Park, Ryo Saegusa, and Shuji Hashimoto, "Autonomous navigation of a mobile robot based on passive RFID," IEICE Transactions on Fundamentals of Electronics, Communications and Computer Sciences Vol.J90-A, pp.901-909, 2007.

[26] T.Tsukiyama, "Navigation System for the Mobile Robots using RFID Tags," IEEE Conference on advanced Robotics, 2003.

[27] Sunhong Park, Ryo Saegusa, and Shuji Hashimoto, "Autonomous navigation of a mobile robot based on passive RFID(2)," The 25th Annual Conference of the Robotics Society of Japan(RSJ), 3D17, pp. 229, 2007. 